\title{
Optimization of immunotherapy for patients with hepatobiliary cancer
}

\author{
Yuji Eso, Hiroshi Seno \\ Department of Gastroenterology and Hepatology, Graduate School of Medicine, Kyoto University, Kyoto, Japan \\ Correspondence to: Dr. Yuji Eso, MD, PhD. Department of Gastroenterology and Hepatology, Graduate School of Medicine, Kyoto University, 54 \\ Shogoin-Kawaharacho, Sakyo-ku, Kyoto 6068507, Japan. Email: yujieso@kuhp.kyoto-u.ac.jp. \\ Comment on: Sahara K, Farooq SA, Tsilimigras DI, et al. Immunotherapy utilization for hepatobiliary cancer in the United States: disparities among \\ patients with different socioeconomic status. Hepatobiliary Surg Nutr 2020;9:13-24.
}

Submitted May 22, 2021. Accepted for publication Jun 03, 2021.

doi: 10.21037/hbsn-21-207

View this article at: https://dx.doi.org/10.21037/hbsn-21-207

Immunotherapy with immune checkpoint inhibitors (ICIs) has been shown its benefit for patients with various types of cancers $(1,2)$. Sahara et al. recently reported the utilization of immunotherapy in the United States among patients with hepatobiliary cancer in Hepatobiliary Surgery and Nutrition (3). In recent years, immunotherapy has become an increasingly popular choice for cancer patients. In practice, however, the choice is biased not only by the progression of the tumor stage, but also by patients' socioeconomic status. In other words, the choice of immunotherapy has not been sufficiently optimized.

There are two strategies crucial for the optimization of immunotherapy. The first strategy is biomarkerbased stratification of patients for optimal response to immunotherapy. Recent studies revealed that tumor microsatellite instability (MSI) status and tumor mutation burden (TMB) contribute significantly to the response to ICIs $(1,4)$, which lead to the approval of the antiprogrammed cell death protein 1 (PD-1) monoclonal antibody (mAb) pembrolizumab for the treatment of advanced or recurrent MSI-high solid tumors that progress after conventional chemotherapies in 2017, and for the treatment of patients with unresectable or metastatic TMBhigh $[\geq 10$ mutations/mega base (Mut/Mb)] solid tumors in 2020. However, the rate of MSI-high hepatobiliary cancer is remarkably low $[0-2.9 \%$ in hepatocellular carcinoma (HCC) and $0-3 \%$ in biliary tract cancer] (4). In addition, recent studies reported that median TMB in HCC was 2.6-5.48 Mut/Mb with $95 \%$ of cases having a TMB of $<10 \mathrm{Mut} / \mathrm{Mb}$, which indicates the limited value of TMB as a predictor of response to immunotherapy (5-7). Regarding the expression of programmed death ligand 1 (PD-L1), unlike other solid tumors, a definite correlation between PD-L1 expression in tumor cells and responses to ICIs has not been demonstrated in HCC; however, PDL1 expression on tumor-associated macrophages may be helpful as indicators of immunotherapy for $\operatorname{HCC}(8,9)$.

The second strategy is to convert "immune-cold" tumors, which do not respond well to immunotherapy, into "immune-hot" tumors. Recently, Pfister et al. reported the possibility of limited efficacy of immunotherapy for nonalcoholic steatohepatitis (NASH)-associated HCC (10). In mouse models mimicking NASH-induced HCC, antiPD-1 therapy expanded activated CD8+PD1+ T cells within tumors but did not achieve anti-tumor effects, which indicates that tumor immune surveillance was impaired in patients with NASH. In addition, a metaanalysis of three randomized phase III clinical trials of antiPD-1 or anti-PD-L1 mAb for advanced HCC revealed that immunotherapy did not improve overall survival (OS) in patients with non-viral HCC \{hazard ratio (HR) $[95 \%$ confidence interval $(\mathrm{CI})]=0.77(0.63-0.94)$ in viral HCC vs. 0.92 (0.77-1.11) in non-viral HCC (10). When the results of each clinical trial are examined in detail, in CheckMate-459 trial evaluating anti-PD-1 mAb nivolumab versus sorafenib as a first-line treatment in patients with unresectable HCC, median OS in non-viral HCC patients was 16.0 months in nivolumab vs. 17.4 months in sorafenib [HR $(95 \% \mathrm{CI})=0.91(0.72-1.16)]$, while those in hepatitis C virus (HCV)-HCC and hepatitis B virus (HBV)-HCC were 
17.5 months in nivolumab $v s .12 .7$ months in sorafenib [HR $(95 \% \mathrm{CI})=0.72(0.51-1.02)]$ and 16.1 months in nivolumab vs. 10.4 months in sorafenib [HR $(95 \%$ CI $)=0.79(0.59-$ 1.07)], respectively, suggesting that non-viral HCC may be less likely to respond to immunotherapy than viral HCC. In KEYNOTE-240 trial evaluating anti-PD-1 mAb pembrolizumab versus placebo, HBV-HCC patients in pembrolizumab arm showed significantly longer OS than those in placebo arm [HR $(95 \% \mathrm{CI})=0.78(0.61-$ 1.00)], while there was no cleat significant difference in OS between the two arms in non-viral HCC patients $[$ HR $(95 \% \mathrm{CI})=0.88(0.64-1.20)]$. On the other hand, in CheckMate-040 trial evaluating nivolumab plus anti-cytotoxic $\mathrm{T}$ lymphocyte antigen 4 (CTLA-4) mAb ipilimumab, there was no difference in median OS among the three groups of HBV-HCC, HCVHCC, and non-viral HCC [14.8 (9.1-20.2) months, 18.8 (11.2-30.8) months, and 15.1 (11.7-18.9) months, respectively]. In IMbrave 150 study evaluating anti-PD-L1 $\mathrm{mAb}$ atezolizumab + anti-vascular endothelial growth factor (VEGF) mAb bevacizumab versus sorafenib, the advantage of atezolizumab + bevacizumab in OS was not clear in non-viral HCC patients [median OS: $17.0 \mathrm{vs}$. 18.1 months; HR $(95 \% \mathrm{CI})=1.05(0.68-1.63)]$, unlike HBVHCC patients [19.0 vs. 12.4 months; HR $(95 \% \mathrm{CI})=0.58$ (0.40-0.83)] and HCV-HCC patients [24.6 vs. 12.6 months; HR $(95 \% \mathrm{CI})=0.43(0.25-0.73)]$. However, in arm F of G030140 study evaluating atezolizumab + bevacizumab versus atezolizumab monotherapy, median progression-free survival in atezolizumab monotherapy group $(n=59)$ was 3.4 months, whereas that in atezolizumab + bevacizumab group $(\mathrm{n}=60)$ was 6.3 months [HR $(80 \% \mathrm{CI})=0.49(0.26-0.92)]$, which may suggest that anti-VEGF mAb may have an add-on effect to ICI monotherapy. In fact, recent studies have revealed the synergistic effects of anti-VEGF $\mathrm{mAb}$ and ICI (11). Anti-VEGF therapy increases intra-tumoral infiltration and survival of cytotoxic $\mathrm{T}$ cells through normalizing vascularization and modulating the immune microenvironment, thus acting synergistically with ICIs. Furthermore, recent studies have shown that thymocyte selection-associated high mobility group box protein (TOX) plays a pivotal role in developing and maintaining exhausted T cells. TOX reduces PD-1 degradation and promotes PD-1 translocation to the cell surface, thus maintaining high PD-1 expression at the cell surface of T cells (11). A recent report revealed that VEGF drives TOX-dependent $\mathrm{T}$ cell exhaustion; therefore, the combined blockade of PD-1/PD-L1 and VEGF pathways could synergistically restore the anti-tumor function of $\mathrm{T}$ cells.

Taken together, although it should be noted that the non-viral HCC group in these clinical trials described so far included not only NASH-HCC but also other populations such as alcohol-related HCC, NASH-HCC may be an "immune-cold" cancer that does not respond well to ICI monotherapy, but the combination of anti-VEGF mAb with ICI could turn it into an "immune-hot" cancer. In order to identify factors that predict response to immunotherapy, and to clarify whether immunotherapy is less effective in pure NASH-HCC than in viral-HCC, and whether anti-VEGF $\mathrm{mAb}$ or anti-CTLA- $4 \mathrm{mAb}$ in combination with anti-PD-1/ $\mathrm{PD}-\mathrm{L} 1 \mathrm{mAb}$ can rescue it, it is necessary to accumulate and analyze real-world clinical data in the future.

\section{Acknowledgments}

Funding: This study was supported by the Ministry of Education, Culture, Sports, Science and Technology (MEXT) KAKENHI Grant Number 21K07912.

\section{Footnote}

Provenance and Peer Review: This article was commissioned by the editorial office of Hepatobiliary Surgery and Nutrition. The article did not undergo external peer review.

Conflicts of Interest: Both authors have completed the ICMJE uniform disclosure form (available at https://hbsn. amegroups.com/article/view/10.21037/hbsn-21-207/coif). Dr. YE reports support from the Ministry of Education, Culture, Sports, Science and Technology (MEXT) KAKENHI, Grant Number 21K07912. The other author has no conflicts of interest to declare.

Ethical Statement: The authors are accountable for all aspects of the work in ensuring that questions related to the accuracy or integrity of any part of the work are appropriately investigated and resolved.

Open Access Statement: This is an Open Access article distributed in accordance with the Creative Commons Attribution-NonCommercial-NoDerivs 4.0 International License (CC BY-NC-ND 4.0), which permits the noncommercial replication and distribution of the article with the strict proviso that no changes or edits are made and the original work is properly cited (including links to both the formal publication through the relevant DOI and the license). 
See: https://creativecommons.org/licenses/by-nc-nd/4.0/.

\section{References}

1. Eso Y, Seno H. Current status of treatment with immune checkpoint inhibitors for gastrointestinal, hepatobiliary, and pancreatic cancers. Therap Adv Gastroenterol 2020;13:1756284820948773.

2. Nakano S, Eso Y, Okada H, et al. Recent Advances in Immunotherapy for Hepatocellular Carcinoma. Cancers (Basel) 2020;12:775.

3. Sahara K, Farooq SA, Tsilimigras DI, et al. Immunotherapy utilization for hepatobiliary cancer in the United States: disparities among patients with different socioeconomic status. Hepatobiliary Surg Nutr 2020;9:13-24.

4. Eso $Y$, Shimizu T, Takeda $\mathrm{H}$, et al. Microsatellite instability and immune checkpoint inhibitors: toward precision medicine against gastrointestinal and hepatobiliary cancers. J Gastroenterol 2020;55:15-26.

5. Wong CN, Fessas P, Dominy K, et al. Qualification of tumour mutational burden by targeted next-generation sequencing as a biomarker in hepatocellular carcinoma. Liver Int 2021;41:192-203.

6. Ang C, Klempner SJ, Ali SM, et al. Prevalence of

Cite this article as: Eso Y, Seno H. Optimization of immunotherapy for patients with hepatobiliary cancer. HepatoBiliary Surg Nutr 2021;10(5):717-719. doi: 10.21037/ hbsn-21-207 established and emerging biomarkers of immune checkpoint inhibitor response in advanced hepatocellular carcinoma. Oncotarget 2019;10:4018-25.

7. Spahn S, Roessler D, Pompilia R, et al. Clinical and Genetic Tumor Characteristics of Responding and NonResponding Patients to PD-1 Inhibition in Hepatocellular Carcinoma. Cancers (Basel) 2020;12:3830.

8. Park DJ, Sung PS, Lee GW, et al. Preferential Expression of Programmed Death Ligand 1 Protein in TumorAssociated Macrophages and Its Potential Role in Immunotherapy for Hepatocellular Carcinoma. Int J Mol Sci 2021;22;4710.

9. El-Khoueiry AB, Sangro B, Yau T, et al. Nivolumab in patients with advanced hepatocellular carcinoma (CheckMate 040): an open-label, non-comparative, phase 1/2 dose escalation and expansion trial. Lancet 2017;389:2492-502.

10. Pfister D, Núñez NG, Pinyol R, et al. NASH limits antitumour surveillance in immunotherapy-treated HCC. Nature 2021;592:450-6.

11. Eso Y, Seno H. Synergistic effects of anti-angiogenesis and immune checkpoint blockade - a new era of systemic chemotherapy for hepatocellular carcinoma. Hepatobiliary Pancreat Dis Int 2021. doi: 10.1016/j.hbpd.2021.04.004. 